\title{
Placental changes in hypertensive pregnancy: a comparison with normotensive pregnancy
}

\author{
Jashan Chhatwal $^{1 *}$, Dev Nanda Chaudhary ${ }^{2}$, Neena Chauhan ${ }^{3}$
}

\begin{abstract}
${ }^{1}$ Department of Obstetrics and Gynecology, Swami Rama Himalayan University, Dehradun, Uttarakhand, India ${ }^{2}$ Department of Obstetrics and Gynecology, GS Medical College, Hapur, Uttar Pradesh, India

${ }^{3}$ Department of Pathology, Swami Rama Himalayan University, Dehradun, Uttarakhand, India
\end{abstract}

Received: 18 February 2018

Accepted: 17 March 2018

\section{*Correspondence:}

Dr. Jashan Chhatwal,

E-mail: dr.jashanchhatwal@gmail.com

Copyright: (c) the author(s), publisher and licensee Medip Academy. This is an open-access article distributed under the terms of the Creative Commons Attribution Non-Commercial License, which permits unrestricted non-commercial use, distribution, and reproduction in any medium, provided the original work is properly cited.

\section{ABSTRACT}

Background: Hypertensive pregnancy may be responsible for vascular damage, enhanced systemic inflammation and insulin resistance in the placenta as oxygen and nutrient transfer is impaired and oxidative stress is generated affecting the placental growth and development. Placental growth pattern in hypertensive pregnancies shows a variable pattern owing to placental insufficiency. Present study was done to investigate the morphological and histological changes in placenta in hypertensive pregnancy.

Methods: A total of 42 pregnant women with hypertensive disorder with gestational age $28-42$ weeks and singleton pregnancy were enrolled as cases in the study. A total of 42 matched normotensive pregnant women were enrolled as controls. All the women were followed up till delivery. At delivery, placental specimen were collected and assessed for morphological, morphometric and histological changes. Findings were compared with normotensives. Data was compared using Independent sample's ' $t$ '-test and Chi-square test.

Results: Mean age of cases was $27.60 \pm 4.37$ years, majority were gravida $1 / 2(66.7 \%), 45.2 \%$ had moderate to severe edema, $50 \%$ had urinary albumin levels $>100 \mathrm{mg} / \mathrm{dl}$. A total of $8(19.0 \%)$ had gestational hypertension, $16(38.1 \%)$ had preeclampsia, $10(23.8 \%)$ had severe preeclampsia and $8(19.0 \%)$ had eclampsia. Mean placental weight and diameter of cases was significantly higher than that of control group. Mean placental thickness was also higher but difference was not significant statistically. Calcification, infarction and hematoma were seen in $45.2 \%, 16.7 \%$ and $11.9 \%$ of cases as compared to $28.6 \%, 4.97 \%$ and $0 \%$ of controls. Histologically, syncytial knots, cytotrophoblastic cellular proliferation, hyalinized area, proliferation of medium sized blood vessels, stromal fibrosis and fibrinoid necrosis in significantly higher proportion of cass as compared to controls $(\mathrm{p}<0.05)$. Mean fetoplacental ratio was $5.01 \pm 0.99$ and $5.24 \pm 0.61$ in controls $(\mathrm{p}=0.195)$.

Conclusions: Hypertension during pregnancy affects the placental growth and development.

Keywords: Hypertension in pregnancy, Histopathology, Morphometry, Placental morphology, Preeclampsia

\section{INTRODUCTION}

Globally, nearly $10 \%$ of all pregnancies are complicated by hypertension and is responsible for a huge burden of maternal as well as prenatal morbidity and mortality. ${ }^{1-3}$ It is also estimated that pregnancy induced hypertension $(\mathrm{PIH})$, one of the hypertensive disorders of pregnancy, affects about $5-8 \%$ of all pregnant women worldwide. ${ }^{4}$ Among pregnancies complicated by hypertenstion a number of structural, morphological and vascular changes take place in placenta during pregnancy. ${ }^{5-7}$ The complications of hypertensive disorders in pregnancy have been attributed to abnormalities in the placenta. ${ }^{8,9} \mathrm{It}$ has been shown that placental villous angiogenesis could 
be responsible for normal perinatal development and survival. ${ }^{10}$

Keeping in view the fact that placental changes and variations have an important impact on fetal well being and pregnancy outcome and these changes are affected by the hypertensive status of the pregnant woman, hence it is important to study as to how and to what extent the hypertensive status affects the placenta morphologically and histopathologically, and in turn how do these changes affect the maternal and fetal outcomes. Hence, the present study was planned with an aim to compare the morphological and histopathological changes seen in placentae of hypertensive mothers from the normotensive mothers and to correlate the findings with maternal and fetal outcomes.

\section{METHODS}

The study was carried out in the Department of Obstetrics and Gynaecology in collaboration with the Department of Pathology, Himalayan Institute of Medical Sciences (HIMS), Swami Ram Nagar, Doiwala, Dehradun, over a period of 12 months. Written informed consent was taken in Hindi and English. Subjects were recruited from the obstetric ward and labour room of SRHU campus, Dehradun.

A total of 42 pregnant women with hypertensive disorder with gestational age 28-42 weeks and singleton pregnancy were enrolled as cases in the study. A total of 42 matched normotensive pregnant women were enrolled as controls. Sample size was calculated on the basis of study by Nahar et al who observed difference in placental weight between hypertensive and normotensive mothers to be 58 gram. ${ }^{11}$

Taking a standard deviation of 90 gram and after making contingency provision of $10 \%$, the calculated sample size at $80 \%$ power and $95 \%$ confidence was 42 . The cases included women having gestational hypertension, preeclampsia, eclampsia, superimposed preeclampsia, chronic hypertension falling in 24-42weeks of pregnancy and having singleton pregnancy.

Women with diabetes mellitus, hypothyroidism, abruptio placentae, jaundice or intrauterine infection (TORCH positive) were excluded from the study. Age, obstetric history, gestational age at enrolment were noted. Assessment of gestational age was done from the last menstrual period.

At the time of enrolment, blood pressure of the subjects was noted and clinical signs of hypertension, viz. edema, facial puffiness were noted. In case of any such sign, duration of problem was noted. All the women underwent urine albumin assessment. Nature and type of hypertensive disorder was determined.
Hypertension was classified and graded as follows as per the ACOG guidelines (Source: Hypertension in Pregnancy, ACOG, 2014):

- Gestational Hypertension: Blood pressure >140/90 presenting after 20 weeks of pregnancy without significant proteinuria.

- Preeclampsia: Blood pressure >140/90 presenting after 20 weeks of pregnancy with significant proteinuria $(>30 \mathrm{mg} / \mathrm{ml}$, or $>300 \mathrm{mg} /$ day or at least $1 \mathrm{~g} / \mathrm{L}[2+]$ on dipstick testing).

- Severe Preeclampsia: Blood pressure >160/110 presenting after 20 weeks of pregnancy with significant proteinuria $(>30 \mathrm{mg} / \mathrm{ml}$, or $>300 \mathrm{mg} /$ day or at least $1 \mathrm{~g} / \mathrm{L}[2+]$ on dipstick testing) with symptomatic manifestation.

- Eclampsia: Eclampsia was defined as the presence of new-onset grand mal seizures in a woman with preeclampsia. Eclampsia can occur before, during or after labour.

All the women were followed up till delivery. Mode of delivery was noted. In case of cesarean delivery, indication for cesarean section was noted. Birth weight of baby, Apgar score at 1 and 5 min were noted. Events like IUGR, stillbirth, NICU admission and neonatal death were noted. Intraoperatively, any special findings were looked for. Immediately after delivery, the placenta along with attached membranes and umbilical cord was collected, washed in running tap water, labeled and then fixed in $10 \%$ formalin.

Gross and microscopic examination of the placenta was carried out. Parameters like placental weight, diameter and thickness, attachment of umbilical cord over placenta, any other structural deviations of placenta or umbilical cord were noted. Morphological and histopathological features like infarction, calcification, hematoma, syncitial knot formation, cytotrophoblastic cell proliferation, hyalinization, proliferation of blood vessels, stromal fibrosis and fibrinoid necrosis were noted.

\section{Statistical analysis}

Data was analyzed using Statistical Package for Social Sciences (SPSS) version 21.0. Chi-square test and Independent samples ' $t$ ' test was used to compare the data. A ' $p$ ' value less than 0.05 indicated a statistically significant association.

\section{RESULTS}

Mean age of cases was $27.60 \pm 4.37$ years, majority were gravida $1 / 2(66.7 \%), 45.2 \%$ had moderate to severe edema, $50 \%$ had urinary albumin levels $>100 \mathrm{mg} / \mathrm{dl}$ (Table 1). 
Table 1: Age, parity and blood pressure at enrolment of the hypertensive and normotensive patients.

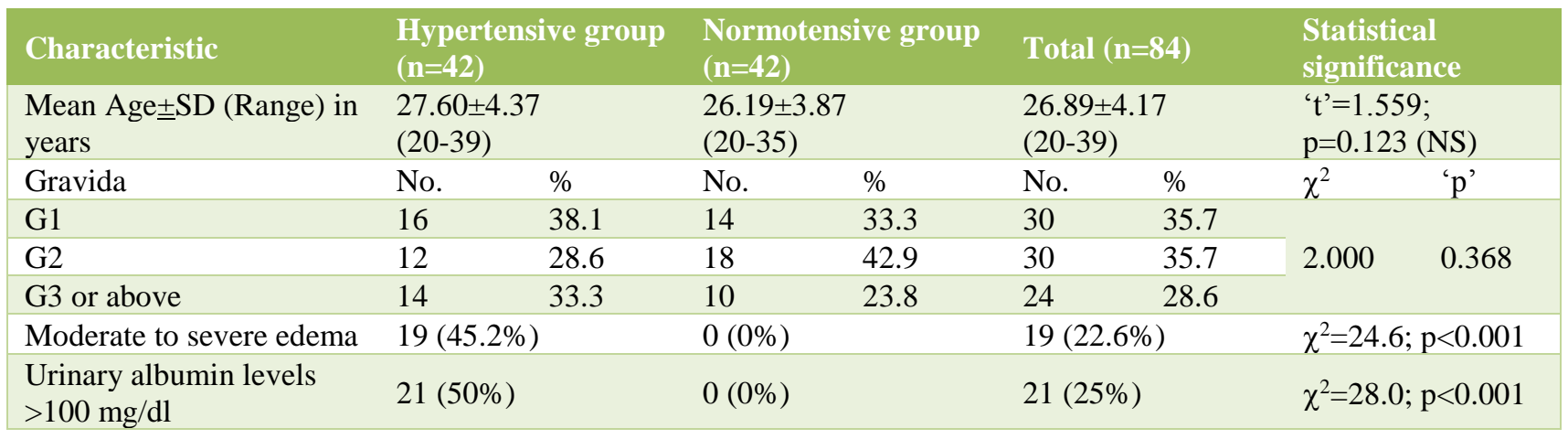

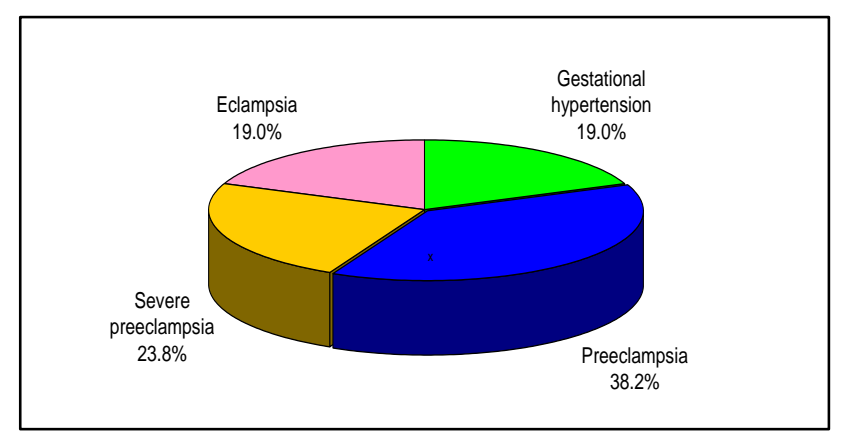

Figure 1: Distribution of cases according to type of hypertensive disorder.

A total of $8(19.0 \%)$ had gestational hypertension, 16 $(38.1 \%)$ had preeclampsia, $10(23.8 \%)$ had severe preeclampsia and $8(19.0 \%)$ had eclampsia (Figure 1).
Mean placental weight and diameter of cases was significantly higher than that of control group. Mean placental thickness was also higher but difference was not significant statistically.

Calcification, infarction and hematoma were seen in $45.2 \%, 16.7 \%$ and $11.9 \%$ of cases as compared to $28.6 \%$, $4.97 \%$ and $0 \%$ of controls.

Histologically, syncytial knots, cytotrophoblastic cellular proliferation, hyalinized area, proliferation of medium sized blood vessels, stromal fibrosis and fibrinoid necrosis in significantly higher proportion of cass as compared to controls $(\mathrm{p}<0.05)$.

Mean fetoplacental ratio was $5.01 \pm 0.99$ and $5.24 \pm 0.61$ in controls $(\mathrm{p}=0.195)$ (Table 2).

Table 2: Comparison of placental morphometric, morphological and histopathological parameters in hypertensive and normotensive groups.

\begin{tabular}{|c|c|c|c|}
\hline Parameter & $\begin{array}{l}\text { Hypertensive group } \\
(\mathrm{n}=\mathbf{4 2})\end{array}$ & $\begin{array}{l}\text { Normotensive group } \\
(\mathrm{n}=\mathbf{4 2})\end{array}$ & Statistical significance \\
\hline \multicolumn{4}{|l|}{ Morphometric } \\
\hline Mean Placental weight \pm SD (Range) in gm & $429.52 \pm 99.06$ & $504.29 \pm 90.12$ & ' $\mathrm{t}$ ' $=3.618 ; \mathrm{p}=0.001$ \\
\hline Mean placental diameter \pm SD (Range) in $\mathrm{cm}$ & $15.23 \pm 2.93$ & $18.63 \pm 4.25$ & $' \mathrm{t} '=4.276 ; \mathrm{p}<0.001$ \\
\hline Placental thickness \pm SD (Range) in $\mathrm{cm}$ & $1.91 \pm 0.47$ & $2.10 \pm 0.52$ & $' t '=1.806 ; p=0.075$ \\
\hline \multicolumn{4}{|l|}{ Morphological } \\
\hline Placental infarction & $7(16.9 \%)$ & $1(2.4 \%)$ & $\chi^{2}=4.97 ; p=0.026$ \\
\hline Calcification & $19(45.2 \%)$ & $12(28.6 \%)$ & $\chi^{2}=2.51 ; p=0.113$ \\
\hline Hematoma & $5(11.9 \%)$ & $0(0 \%)$ & $\chi^{2}=5.32 ; p=0.021$ \\
\hline \multicolumn{4}{|l|}{ Histopathological } \\
\hline Syncytial knot & $38(90.5 \%)$ & $26(61.9 \%)$ & $\chi^{2}=9.45 ; p=0.002$ \\
\hline Cytotrophoblastic cellular proliferation & $30(71.4 \%)$ & $8(19.0 \%)$ & $\chi^{2}=23.3 ; p<0.001$ \\
\hline Hyalinized areas & $26(61.9 \%)$ & $5(11.1 \%)$ & $\chi^{2}=22.5 ; \mathrm{p}<0.001$ \\
\hline Proliferation of medium sized blood vessels & $26(61.9 \%)$ & $6(14.3 \%)$ & $\chi^{2}=20.2 ; p<0.001$ \\
\hline Stromal fibrosis & $11(26.2 \%)$ & $1(2.4 \%)$ & $\chi^{2}=9.72 ; p<0.001$ \\
\hline Fibrinoid necrosis & $35(83.3 \%)$ & $12(53.4 \%)$ & $\chi^{2}=9.22 ; p<0.001$ \\
\hline \multicolumn{4}{|l|}{ Feto-placental ratio (FPR) } \\
\hline Mean FPR \pm SD (Range) & $5.01 \pm 0.99(2.9-7.2)$ & $5.24 \pm 0.61(4.2-6.6)$ & $' t '=1.705 ; p=0.195$ \\
\hline
\end{tabular}




\section{DISCUSSION}

In present study, mean placental weight of pregnancies complicated by hypertension was 429.52 gms which was significantly lower as compared to 504.29 gms for normotensive cases. Mean placental diameter was also significantly lower in hypertensive cases $(15.23 \mathrm{~cm})$ as compared to that in normotensive cases (18.63). Although, mean placental thickness was also lower (1.90 $\mathrm{cm}$ ) in hypertensive cases as compared to that in normotensive cases (2.10) yet this difference was not significant statistically. Placental morphometry parameters in hypertensive pregnancies have been reported to be affected as compared to normotensive pregnancies in different studies.

In different studies, placental weight in hypertensive pregnancies has been reported to vary from $345.96 \mathrm{~g}$ to $524.24 \mathrm{~g}$ whereas that in normotensive pregnancies ranged from $435.92 \mathrm{~g}$ to $557.8 \mathrm{~g} .{ }^{12,13}$ The difference in placental weight of two groups in present study was $77.77 \mathrm{~g}$. Extent of this difference has ranged widely in different studies. Qureshi et al found this difference to be $33 \mathrm{~g}$ only while Motwani et al found it as $67 \mathrm{~g}$, similar to our study, Kulandaivelu et al in their study reported it to be $73 \mathrm{~g}$, however, on a higher note Nag et al found this difference to be as high as $94 \mathrm{~g}$ and Salmani et al even surpassed this and reported this difference as $124 \mathrm{~g} .{ }^{13-17}$ The profile of differences in placental weight between hypertensive group and normotensive groups in different studies might be due to difference in different types of HDPs included in the assessment. Studies reporting a higher difference generally have a higher component of severe HDPs like severe preeclampsia and eclampsia as compared to gestational hypertension.

With respect to placental diameter too, various studies have found it to be lower in hypertensive cases as compared to normotensive cases. In present study, we found a difference of $3.4 \mathrm{~cm}$ between hypertensive and normotensive cases. However, Kulandaivelu et al found this difference to be only $1.06 \mathrm{~cm}$, Qureshi et al found this difference to be $1.6 \mathrm{~cm}$, while Singh and Gugapriya found this difference to be somewhat close to that reported in present study at $2.49 \mathrm{~cm}^{13,15,18}$ On the other hand, Ranga SS et al reported it as $5 \mathrm{~cm}$. Porwal et al. in their study reported this difference in terms of proportion of women with placental diameter $<20 \mathrm{~cm}$ to be $90 \%$ in hypertensive group as compared to $63.33 \%$ in normotensive group. ${ }^{19,20}$ Thus, this explains the extent of difference in terms of proportional differences.

As far as placental thickness in concerned, considerable variability in diameter has been reported in different studies. Kulandaivelu et al.15 reported the placental diameter of hypertensive women to be $1.23 \mathrm{~cm}$ as compared to $1.42 \mathrm{~cm}$ in normotensive women (difference $0.19 \mathrm{~cm}$ ), while Qureshi et al reported them as 2.2 and 3 $\mathrm{cm}$ (diff. $0.8 \mathrm{~cm}$ ), Singh and Gugapriya reported them as 2.39 and $2.77 \mathrm{~cm}$ (diff. $0.38 \mathrm{~cm}$ ) while Ranga $\mathrm{SS}$ et al noted them as $1.9 \mathrm{~cm}$ and $2.4 \mathrm{~cm}$ (diff. $0.5 \mathrm{~cm}$ ). ${ }^{13,15,18,20} \mathrm{In}$ present study, we found them as 1.91 and $2.1 \mathrm{~cm}$ with a difference of $0.2 \mathrm{~cm}$ only. The reason for variance in placental thickness measurements and difference between the two groups in different studies could be owing to difference in method of measurement in different studies. Incidentally, placental thickness is not even throughout the placenta. It is maximum at the centre and minimum in the area between periphery and centre. In present study, in order to avoid a discrepancy, we took average thickness taken at three different points in the three arbitrary chosen locations in placenta. However, in some studies, like the one conducted by Salmani et al, the thickness has been measured at centre and incidentally in their study, the difference in average thickness of hypertensive mothers and normotensive mothers was $0.09 \mathrm{~cm}$ only. ${ }^{17}$ The reason for this could be attributed to the fact that at the centre, umbilical cord is joined at placenta and sometimes the remnants of umbilical cord interfere with the thickness of placenta and hence the results might alter. As such, present study did not find a significant difference in thickness of placenta between hypertensive group and normotensive group. In view of high inconsistency in results in different studies, it is essential that a standard measurement system for measuring thickness of placenta should be adopted.

In present study, among different morphological features, we found placental infarction to be present in $16.7 \%$ of hypertensive cases as compared to $2.4 \%$ normotensive cases, calcification in $45.2 \%$ of hypertensive cases as compared to $28.6 \%$ of normotensive cases and hematoma in $11.9 \%$ of hypertensive group as compared to none of the normotensive group. With respect to placental infarction and hematoma, the differences between two groups were significant statistically too. Similar to results of present study, Akhlaq et al also found proportion of cases with infarct to be higher in hypertensive group $(28 \%)$ as compared to normotensive group (20\%). ${ }^{21}$ The incidence of infarcts in their study was subclassified as red infarcts, white infarcts, jelly infarcts and membrane infarcts and for each of these types the proportion was significantly higher in cases as compared to controls. Although in present study we did not make that many categories, yet we found that infarction was in general significantly higher in hypertensive group as compared to normotensive group. Motwani et al in their study also found incidence of calcification (70\% vs $26.66 \%)$ and infarction $(43.33 \%$ vs $3.3 \%)$ to be higher in hypertensive as compared to normotensive groups. ${ }^{14}$ Qureshi et al in their study found infarction in $53.3 \%$ of hypertensive as compared to none of the normotensive women. ${ }^{13}$ Singh and Gugapriya, similar to present study found incidence of infarction ( $58 \%$ vs $12 \%$ ), hematoma ( $48 \%$ vs $8 \%$ ) and calcification ( $44 \%$ vs $14 \%$ ) and found the difference to be statistically significant for all the three parameters. ${ }^{12}$ Compared to their study, except for calcification, for none of the parameters we found the incidence to be too high. Yet we were able to find statistically significant difference between study and control group for placental 
infarction and hematoma respectively. Other studies like Porwal et al and Range SS, have also found the incidence of infarction, calcification and hematoma formation to be higher in hypertensive group as compared to normotensive group. ${ }^{19,20}$

With respect to histological profile of placenta, the present study found increased syncytial knots in $90.5 \%$ of cases of hypertensive group as compared to only $61.9 \%$ of normotensive group, cytotrophoblastic proliferation in $71.4 \%$ of hypertensive cases as compared to $19 \%$ of normotensive cases, hyalinized areas in $61.9 \%$ of hypertensive cases as compared to $11.9 \%$ of normotensive cases, proliferation of medium sized blood vessels in $61.9 \%$ hypertensive cases as compared to $14.3 \%$ of normotensive cases, stromal fibrosis in $26.2 \%$ of hypertensive cases as compared to $2.4 \%$ of normotensive cases and fibrinoid necrosis in $83.3 \%$ of hypertensive cases as compared to $52.4 \%$ of normotensive cases. For all these findings we found a significant increased incidence in cases of hypertensive group as compared to normotensive group.

Similar to present study, Akhlaq et al reported fibroid necrosis it in $88 \%$ of hypertensive cases and $40 \%$ of normotensive cases. ${ }^{21}$ In other studies too, its prevalence has been reported to range from $80 \%$ to $100 \%$ in hypertensive women. ${ }^{11,15}$ For other major findings such as cytotrophoblastic cellular proliferation which was seen in $71.4 \%$ of cases in hypertensive group and $52.4 \%$ of normotensive cases in present study, we found supportive evidence from Nag et al, Motwani et al and Porwal et al. ${ }^{14,16,19}$ For other major findings too, like hyalinized areas we had $61.9 \%$ hypertensive cases and $11.9 \%$ normotensive cases. Motwani et al in their study reported hyalinized areas in $46.66 \%$ of hypertensive cases and $13.3 \%$ of normotensive cases, which is much lower than that reported in present study. ${ }^{14}$ However, the difference might be owing to difference in evaluation method. In present study we used a more sensitive criteria for hyalinization and reported presence of hyalinization in any of the representative field visualizations instead of taking an average. Proliferation of medium sized vessels was seen in $61.9 \%$ of our cases as compared to $19 \%$ of controls. Motwani et al on the other hand reported it in terms of mean $\%$ as $4.19 \%$ in hypertensive cases and $3.46 \%$ in normotensive cases. ${ }^{14}$ The difference once again could be owing to difference in method of evaluation. In present study we recorded it in categorical terms instead of basing our findings on counts, however, owing to use of these more sensitive criteria obtained a higher positivity rate in hypertensive cases as compared to normotensive cases. But using a similar criteria, Porwal et al found results similar to present study with $83.34 \%$ positivity in hypertensive cases as compared to $0 \%$ in normotensive cases. ${ }^{19}$ Thus use of this criteria was not only more sensitive but also more specific and hence, we can justify the criteria used in our study. In present study, stromal fibrosis was seen in $26.2 \%$ of hypertensive cases as compared to $2.4 \%$ of normotensive cases.
Compared to this Rana et al reported stromal fibrosis in $40 \%$ of hypertensive cases and $15 \%$ of normotensive cases. $^{22}$

Thus, the present study endorsed the reports in previous studies that placental morphometry, morphology and histology is in general adversely affected in hypertensive disorders of pregnancy. Although proportion and spectrum of histological variations in different studies showed a variation, yet it is evident that all the studies report abundance of abnormal variations in placentas of hypertension affected pregnancies. Despite these differences, increased syncytial knots, fibrinoid necrosis, cytotrophoblastic proliferation remain the mainstay of abnormal findings in almost all the studies.

In present study, feto-placental ratio (FPR) ranged from 2.9 to 7.2 with a higher mean value in normotensive cases $(5.24 \pm 0.61)$ as compared to that in hypertensive cases

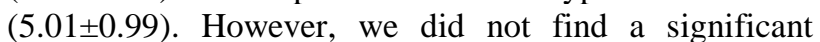
difference in FPR of two groups. Normally, fetoplacental weight ratio varies between 6 and 8 , thus neither cases in two groups could qualify for an ideal FPR. The reason for this could be abundant malnutrition in the pregnant women in our settings. In our settings, most of the pregnant women belonged to lower socio-economic class where malnutrition is quite rampant and this could have affected the FPR in both the groups, more so in pregnancies complicated by hypertension. Similar to present study, Kambale et al. too in their study reported quite close FPR in cases and controls (5.38 and 5.68) and found them to be below ideal ratio as observed in present study. ${ }^{23}$ Ranga et al in their study also observed FPR of 5.5 in cases and 5.6 in controls and did not find a significant difference between two groups. ${ }^{20}$ In different studies reviewed by us, only one study showed FPR value $>6: 1$ for both hypertensive cases (6.7) and normotensive cases (7.2) and found significant difference between two groups, however, this study was carried out in Tamil Nadu, Southern India where rate of malnutrition is relatively lesser as compared to our study area, which owing to its geographical and climatic conditions does not have a flourishing economy and as such is reason for rampant malnutrition among the population. ${ }^{17}$ In fact, in malnourished conditions, the FPR does not follow the standard patterns..

\section{CONCLUSION}

The findings of the present study thus suggest that placental morphology and histopathology is affected in hypertensive pregnancies which might be the reason for placental insufficiency in these cases. How these morphological and histopathological changes affect the maternal and perinatal outcome needs to be studied separately.

Funding: No funding sources

Conflict of interest: None declared 
Ethical approval: The study was approved by the Institutional Ethics Committee

\section{REFERENCES}

1. Dolea C, Abou Zahr C, eds. Global Burden of Hypertensive Disorders of Pregnancy in the Year 2000. Geneva:World Health Organisation;2003.

2. Muti M, Tshimanga M, Notion GT, Bangure D, Chonzi P. Prevalence of pregnancy induced hypertension and pregnancy outcomes among women seeking maternity services in Harare, Zimbabwe. BMC Cardiovasc Disorders. 2015;15:111.

3. Ye C, Ruan Y, Zou L, Li G, Li C, Chen Y, et al. The 2011 Survey on hypertensive disorders of pregnancy (HDP) in China: prevalence, risk factors, complications, pregnancy and perinatal outcomes. PLoS One. 2014 Jun 17;9(6):e100180.

4. Arshad A, Pasha W, Khattak TA, Kiyani RB. Impact of pregnancy induced hypertension on birth weight of newborn at term. 2011;15(2):113-5.

5. Kurdukar MD, Deshpande NM, Shete SS, Zawar MP. Placenta in PIH. Indian J Pathol Microbiol. 2007 Jul; 50(3):493-7.

6. Krielessi V, Papantoniou N, Papageorgiou I, Chatzipapas I, Manios E, Zakopoulos N et al. Placental pathology and blood pressure's level in women with hypertensive disorders in pregnancy. Obstet Gynecol Int. 2012;2012:684083.

7. Soma H, Yoshida K, Mukaida T, Tabushi Y. Morphologic changes in the hypertensive placenta. Contributions Gynecol Obstet. 1982;9:358-75.

8. Myatt L. Role of placenta in preeclampsia. Endocrine. 2002;19(1):103-11.

9. Misra DP, Salafia CM, Miller RK, Charles AK. Nonlinear and gender-specific relationships among placental growth measures and the fetoplacental weight ratio. Placenta. 2009;30(12):1052-7.

10. Kingdom J. Adriana and Luisa Castellucci Award Lecture. Placental pathology in obstetrics: adaptation or failure of the villous tree? Placenta. 1997;19(56):347-51.

11. Nahar L, Nahar K, Hossain MI, Jahan S, Rahman M. Placental changes in pregnancy induced hypertension. Mymensingh Med J. 2013 Oct;22(4):684-93.

12. Singh $\mathrm{S}$ and Gugapriya TS. A cross sectional morphometric study of hypertensive with normal placentae and its correlation with fetal outcome. Int $\mathbf{J}$ Anat Res. 2014;2(2):437-42.
13. Qureshi MA, Bhurgri GR, Yousfani GM. Morphological and histological changes in placenta of hypertensive and gestational diabetic women. Med Formum Monthly. 2014;25(10):10-4.

14. Motwani R, Sontakke Y, Goyal M. Effects of Pregnancy Induced Hypertension on human placenta. JEMDS. 2013;2(33):6275-82.

15. Kulandaivelu AR, Srinivasamurthy BC, Murgan A, Morphology and morphometric study of human placenta in rural southern India. Br J Med Med Res. 2014;4(15):2995-3008.

16. Nag U, Chakravarthy VK, Rao DR. Morphological changes in placenta of hypertensive pregnant women. IJRRMS. 2013;3(2):1-4.

17. Salmani D, Purushothaman S, Somashekara SC, Gnanagurudasan E, Sumangaladevi K, Harikishan R, et al. Study of structural changes in placenta in pregnancy-induced hypertension. J Natural Sci Biol Med. 2014;5(2):352-5.

18. Singh S and Gugapriya TS. A cross sectional morphometric study of hypertensive with normal placentae and its correlation with fetal outcome. Int $\mathbf{J}$ Anat Res. 2014;2(2):437-42.

19. Porwal V, Jain D, Gupta S, Khandelwal S, Kasliwal N. Spectrum of placental changes in pregnancy induced hypertension. Annal Pathol Lab Med. 2017;4(1):A69-A76.

20. Siva Sree Ranga MK, Kumar KV, Thangam A, Vasantha Mallika MC. Morphological variations of human placentae in preterm labor, pregnancyinduced hypertension, and gestational diabetes mellitus. Int J Scientific Study. 2017;4(11):144-9.

21. Akhlaq M, Nagi AH, Yousaf AW. Placental morphology in pre-eclampsia and eclampsia and the likely role of NK cells. Indian J Pathol Microbiol. 2012;55:17-21.

22. Rana S, Diwan Y, Chauhan RS, Diwan D, Gupta A. Comparative study of histology of placenta in normotensive and hypertensive cases. JMSCR. 2017;5(3):18635-40.

23. Kambale T, Iqbal B, Ramraje S, Swaimul K, Salve S. Placental morphology and fetal implications in pregnancies complicated by pregnancy-induced hypertension. Med J DY Patil Univ. 2016;9:341-7.

Cite this article as: Chhatwal J, Chaudhary DN, Chauhan N. Placental changes in hypertensive pregnancy: a comparison with normotensive pregnancy. Int J Reprod Contracept Obstet Gynecol 2018;7:3808-13. 